\title{
Long-term impact of bariatric surgery in diabetic nephropathy
}

\begin{abstract}
Background: Bariatric surgery has been shown to improve and resolve diabetes. However, limited literature about its impact on end-organ complications of diabetes is available. The aim of this study was to examine the long-term effect of bariatric surgery on albuminuria.

Methods: We studied 101 patients with pre-operative diabetes and albuminuria [defined as urine albumin:creatinine ratio $(\mathrm{uACR})>30 \mathrm{mg} / \mathrm{g}$ ] who underwent bariatric surgery at an academic center from 2005 to 2014.

Results: Fifty-seven patients $(56 \%)$ were female with a mean age of $53( \pm 11)$ years. The mean pre-operative BMI and glycated hemoglobin (HbA1c) were $43.1( \pm 7.6) \mathrm{kg} / \mathrm{m} 2$ and $8.4( \pm$ $1.8) \%$, respectively. The median pre-operative uACR was 80.0 (45.0-231.0) $\mathrm{mg} / \mathrm{g}$. Bariatric procedures included Roux-en-Y gastric bypass $(n=75,74 \%)$ and sleeve gastrectomy $(n=26$, $26 \%)$. The mean follow-up period was $61( \pm 29)$ months. At last follow-up, the mean BMI was $33.8( \pm 8.3) \mathrm{kg} / \mathrm{m} 2$. The overall glycemic control improved after bariatric surgery. At last follow-up, $73 \%$ had good glycemic control (HbA1c < 7\%) and 27\% met diabetes remission criteria. The mean HbA1c at last follow-up was $6.7( \pm 1.0) \%$ and the median uACR was 30 (IQR 7-94) mg/g. Albuminuria improved in $77 \%$ and resolved in $51 \%$ of patients at long-term.

Conclusions: Bariatric surgery has a significantly positive impact on albuminuria in patients with obesity and type 2 diabetes. Our data showed almost an $80 \%$ improvement in albuminuria at the short- and long-term period after bariatric surgery.
\end{abstract}

Keyword: Albuminuria; Bariatric surgery; Diabetes; Gastric bypass; Nephropathy; Sleeve gastrectomy 